\title{
Estimación del costo para el diagnóstico precoz de la hidatidosis en niños de 5 y 12 años en la provincia de Ñuble, Región del Bío-Bío, Chile
}

\author{
M. José Farías C. y Alex Medina G.
}

\begin{abstract}
Universidad del Bío-Bío, Chile. Facultad de Ciencias de la Salud y Los Alimentos.

Programa Magíster en Salud Pública (MJFC).

Facultad de Ciencias Empresariales (AMG).

Fuente de financiamiento: Recursos personales de los investigadores y apoyo del Departamento de Gestión Empresarial Facultad de Ciencias Empresariales, Universidad del Bío-Bío.
\end{abstract}

Recibido: 21 de diciembre de 2009 Aceptado: 24 de noviembre de 2010

Correspondencia a: María José Farías Carrasco marifariasc@gmail.com

\section{Introducción}

L a hidatidosis humana se considera como accidente biológico y constituye una enfermedad de pronóstico grave, desde el punto de vista de la enfermedad, por las repercusiones económicas que representa, debido a prolongadas hospitalizaciones, complicaciones post operatorias, tratamientos, alta mortalidad quirúrgica y el elevado costo monetario que tiene su diagnóstico y tratamiento, además del largo ausentismo laboral, pago de subsidios y otros gastos que no son posibles de evaluar con precisión ${ }^{1}$.

Chile tiene una las de mayores tasas de hidatidosis humana en el mundo ${ }^{2}$. Las tasas de incidencia para el período 2001-2005, de 2,2/100.000 habitantes, cifra emanada del sistema de notificación obligatoria del Ministerio de Salud (Chile) es coincidente a lo referido por la OPS, la que muestra que esta tasa se ha mantenido estable desde 1992, con valores entre 2 y 2,5 casos/ 100.000 habitantes. Sin embargo, la aparición anual de casos nuevos indicaría que ésta es una enfermedad cuyo agentes etiológico se encuentra presente en Chile, aun cuando su incidencia se ha mantenido estable. En relación a las tasas de egresos hospitalarios la tasa promedio es de 6,0 casos/100.000 habitantes. Las regiones más comprometidas son Aysén (43,8/100.000 habitantes), La Araucanía (28,1/100.000 habitantes) y Magallanes (16,4/100.000 habitantes $)^{3}$.

La letalidad de la enfermedad alcanza a $3 \%$ y la mortalidad osciló entre 0,3 y $0,4 / 100.000$ habitantes en el 2003; su notificación alcanzó a 280 casos, registrando Bío-Bío, Aysén y Magallanes las tasas más altas. Los egresos hospitalarios por hidatidosis corresponden al $0,07 \%$ del total de egresos del país en los años 2001 y 2002, con 1.144 y 1.118 casos respectivamente ${ }^{4}$.

La hidatidosis es una enfermedad grave que generalmente causa problemas por efecto de masa o infecciones secundarias. Sin embargo, puede causar reacciones alérgicas graves, el quiste hidatídico puede romperse hacia fuera del órgano parasitado dando lugar a múltiples quistes secundarios, puede tener localizaciones peligrosas como en el cerebro, corazón o mediastino y su tratamiento puede requerir medicamentos por un tiempo muy prolongado o intervenciones quirúrgicas con resolución potencialmente fatal $(<2 \%)$, complicaciones postoperatorias $(10-25 \%)$ y recurrencia a largo plazo $(2-10 \%)^{5}$.

El impacto de la hidatidosis en salud pública radica en que es una enfermedad destructora, grave cualquiera sea su localización, disminuye la capacidad de trabajo de quien la padece antes, durante y después del diagnóstico y tratamiento. Además es causa de prolongadas hospitalizaciones, alta frecuencia de complicaciones postoperatorias, alta mortalidad quirúrgica y un elevado costo monetario que implican su diagnóstico y tratamiento ${ }^{6,7}$.

El impacto económico producido por la equinococosis quística, es la suma de varios costos; entre ellos, los gastos para los Servicios de Salud estatales, mortalidad 
y pérdidas por decomisos de vísceras y disminución de productividad. Esto último presenta diferentes grados de dificultad para su estimación exacta, pero diversos estudios demuestran la importancia de su inclusión para el cálculo del impacto real de la enfermedad. La estimación de varios de estos costos, como ha quedado demostrado en numerosos estudios al respecto, sumado a la falta y discontinuidad de la información, hacen necesario elaborar modelos que permitan una estimación de costos lo más acertada posible y reproducible a lo largo del tiempo ${ }^{8}$.

La utilidad y la validez de la ecografía en el diagnóstico de la hidatidosis humana han sido ampliamente verificadas, tanto en pacientes sintomáticos como en asintomáticos, estimándose la sensibilidad y especificidad de este examen en $100 \%$ y 96 a $97 \%$, respectivamente 9 . De las técnicas confirmatorias de la infección actualmente en uso, ELISA IgG es el método de elección por su alta sensibilidad $(86 \%)$ y especificidad $(93 \%)^{10}$ y un valor predictor positivo elevado; los falsos positivos no alcanzan a $3 \%{ }^{11}$.

Desde el punto de vista del equipamiento ecográfico, para un eficiente tamizado rural no es necesario contar con instrumental extremadamente complicado. Incluso el personal no especializado puede ser entrenado para lograr buenos resultados usando ecógrafos portátiles sencillos acompañados de pequeños generadores eléctricos, aun en ausencia de instalaciones sanitarias o electrificación urbana ${ }^{9}$.

Considerando el efecto del tratamiento de esta enfermedad y lo que implica en recursos para los Servicios de Salud estatales como también para la persona afectada y sus familiares, es conveniente detectar tempranamente esta patología mediante un examen preventivo a las personas de mayor riesgo, fundamentalmente los niños de zonas rurales ${ }^{12}$. Este examen consiste en la determinación en suero de anticuerpos tipo IgG específicos (ELISA indirecto) y la realización de una ecotomografía abdominal a cada niño incluido en el grupo de riesgo. Para una mayor efectividad se propone que sea realizado en el último control de niño sano, que es a los 5 años y 11 meses de edad, y posteriormente en el colegio, a la edad de 12 años, lo cual facilita la obtención de muestras 9 .

El objetivo del presente trabajo es estimar los costos para el Servicio de Salud de la Provincia de Ñuble que representaría un programa de diagnóstico precoz de la hidatidosis durante el período de 2006-2007, evaluar el impacto económico generado y así poder crear programas de control para la provincia.

\section{Poblaciones y Métodos}

El universo está constituido por niños de 5 y 12 años durante los años 2006 y 2007. El primer grupo correspon- de al Programa de Salud del Niño del Ministerio de Salud, que consta de la cohorte de 5 años de edad en el control de niño sano y el segundo grupo lo conforman niños de 12 años pertenecientes a colegios de zonas rurales. Para esto se tomaron datos históricos.

Para la recolección de datos se determinaron todas las actividades involucradas en este procedimiento: medición de IgG específica para hidatidosis (ELISA indirecta), la realización de una ecotomografía abdominal y todos los gastos que involucran la toma de muestra y viajes a los colegios para su ejecución. La muestra se toma a todos los niños pertenecientes al Programa de Salud del Niño que tienen hasta 5 años y 11 meses en el último control de niño sano, donde la cobertura en salud en zonas rurales es de $75 \%$, es decir, 4.139 niños en total. Respecto a los niños de 12 años se optó por ellos, debido a que en la enseñanza básica hay una mayor cobertura de la educación y menor deserción por parte de los alumnos de colegios rurales; para este grupo la población de niños fue de 4.903. En suma, el total de niños de 5 y 12 años incluidos en el estudio fue de 9.042. Se optó por elegir estas dos categorías de edades ya que la hidatidosis es una patología que se adquiere en forma temprana y su desarrollo es lento.

Para realizar la evaluación del diagnóstico precoz se utilizaron las estadísticas del INE de la provincia de Ñuble del año 2002, extrayéndose el número de niños de 5 y 12 años de zona rural, a partir de los cuales se hicieron proyecciones. Como las estadísticas son generales (Urbano y Rurales) se procedió según las estimaciones de la evolución porcentual de la población urbana-rural; se estimó en $69 \%$ el crecimiento urbano a través de los años y de acuerdo a esto se hicieron estimaciones de la población rural para los niños de 5 años. Para el cálculo de los niños de 12 años se utilizó la Tasa de Matrícula por Edades Simples.

Para la determinación de los costos se identificaron los costos reales directos incurridos y los costos reales indirectos mediante la metodología de costos basados en actividades (ABC), para lo cual se unificaron todas las actividades que se realizaron en la toma de muestra para la realización del test de ELISA, ecotomografía abdominal $\mathrm{y}$ transporte de los funcionarios a las zonas rurales.

Para determinar los costos se categorizaron los costos directos e indirectos de las intervenciones:

Costos directos: Para este estudio, estaban constituidos por todos los factores que intervienen en el diagnóstico, a saber:

- Mano de obra directa. Fue necesario identificar las categorías o estamentos de personal que intervienen en el proceso de diagnóstico y los tiempos que este factor destina a este proceso. Luego de identificar el recurso humano, éste se valorizó. Para ello se consideró un 
promedio de los salarios de cada categoría de personal, ya que al interior de cada categoría se presentaban diferencias en los salarios (responsabilidad, profesional, antigüedad y otras).

- Equipos, instrumentos y muebles de uso directo. Corresponden a todos los elementos que están disponibles donde se realizó la intervención.

- Insumos. Correspondían a los insumos directos utilizados en el diagnóstico.

Costos indirectos: Son aquellos que, aunque se relacionan con la intervención, no son directamente medibles y/o fácilmente asociables a la intervención que se costeó. Como por ejemplo, gastos en agua y luz eléctrica, etc. Por lo que estos costos se distribuyeron de acuerdo a los conductores de costos, mediante la utilización de la metodología de costos $\mathrm{ABC}$.

\section{Resultados}

A continuación se muestran las diferentes técnicas para los procedimientos del diagnóstico precoz de la enfermedad.

\begin{tabular}{|c|c|}
\hline & Provincia de Ñuble (\$) \\
\hline Extracción de sangre & 2.643 \\
\hline Ecotomografía & 9.141 \\
\hline ELISA indirecto & 8.926 \\
\hline Total & 20.710 \\
\hline
\end{tabular}

- Diagnóstico precoz en niños de 5 años. Se consideró el último control de niño sano, en el respectivo consultorio, lo que implicó la extracción de sangre, para el examen de ELISA indirecto y la realización de una ecotomografía abdominal (este examen es realizado por un especialista en el consultorio), por lo que el costo por diagnóstico a cada niño de 5 años es de $\$ 20.710$, por atención en el respectivo consultorio (Tabla 1).

- Diagnóstico precoz en niños de 12 años. Este grupo incluyó a niños pertenecientes a los colegios de zonas rurales. Estos exámenes se estimaron ser realizados en el mismo establecimiento. En este caso la toma de la ecotomografía puede ser realizada por una enfermera la cual es instruida para poder llevar a cabo este examen. Para la realización del examen de ELISA indirecto se utiliza la misma Tabla 1, debido a que la muestra es llevada a un laboratorio.

Para la determinación del costo de extracción de sangre (Tabla 2) se consideró que el personal de salud se trasladaba al establecimiento educacional, por lo que en este caso solamente se tomaron en cuenta los costos directamente relacionados a la extracción. En lo que respecta a la ecotomografía abdominal, ésta se toma en el mismo establecimiento con un equipo portátil (Tabla 2).

Considerando que el personal de salud se desplazó al recinto educacional, se incorporó el transporte de los funcionarios a los recintos escolares; para Chillán, en un día se estimó la atención a 24 niños, por lo tanto, el viaje se dividió por los alumnos atendidos, o sea $\$ 862$ por estudiante. Este mismo procedimiento se realizó con las otras ciudades según tiempo de llegada y cuántos alumnos se atendieron en el día, por lo que el costo por transporte se muestra en la Tabla 3, distribuido por cada una de las zonas de la provincia.

\begin{tabular}{|c|c|c|c|c|c|}
\hline \multirow[t]{2}{*}{ Item } & \multicolumn{2}{|c|}{ Niños de 5 años } & \multicolumn{2}{|c|}{ Niños de 12 años } & \multirow[b]{2}{*}{$\begin{array}{l}\text { Examen ELISA } \\
\text { indirecto (\$) }\end{array}$} \\
\hline & $\begin{array}{c}\text { Técnica de extracción } \\
\text { de sangre (\$) }\end{array}$ & $\begin{array}{l}\text { Ecotomografía } \\
\text { abdominal (\$) }\end{array}$ & $\begin{array}{c}\text { Técnica de extracción } \\
\text { de sangre (\$) }\end{array}$ & $\begin{array}{l}\text { Ecotomografía } \\
\text { abdominal (\$) }\end{array}$ & \\
\hline Recurso humano & 885 & 5.090 & 885 & 1.260 & 2.871 \\
\hline Equipos, instrumento y muebles & 35 & 492 & 4 & 112 & 2.168 \\
\hline Insumos & 536 & 417 & 386 & 73 & 1.089 \\
\hline Alimentación & 285 & 2.014 & 282 & 760 & 900 \\
\hline Administración & - & 5 & - & - & 28 \\
\hline Consumos básicos y calefacción & 900 & 830 & - & - & 1.400 \\
\hline Depreciación de establecimiento & 2 & 293 & - & - & 470 \\
\hline Total (\$) & 2.643 & 9.141 & 1.557 & 2.205 & 8.926 \\
\hline
\end{tabular}




\section{Determinación de costos}

En la estimación del costo de los exámenes para el diagnóstico precoz de la hidatidosis, en la cohorte de niños de 5 años se incluyó a la totalidad debido a que el control de salud es universal. En el grupo de niños de 12 años se utilizaron las tasas de matriculas para proyectar cuántos niños se realizarían el examen.

- Niños de 5 años. En la Tabla 1 se aprecia el costo del diagnóstico precoz en niños de 5 años. Una vez obtenido el valor correspondiente a cada una de las actividades y recursos necesarios, el costo total para la provincia de Ñuble en el diagnóstico precoz superó los $\$ 85.000 .000$ (US aproximado: 175.000 a la fecha de publicación del artículo) (Tabla 4).

- Niños de 12 años. En lo que respecta a este grupo, el costo de transporte involucrado en el diagnóstico precoz se muestra en la Tabla 5, debidamente distribuido por ciudad. Una vez obtenido el costo de transporte, se determinó el costo total por diagnóstico, tal como se muestra en la Tabla 6 , costo que es diferente según la ciudad en donde éste se realice.

Con la información obtenida del diagnóstico en niños de 5 años y en niños de 12 años, fue posible establecer el costo total para la provincia de Ñuble y que se expone en la Tabla 7. Como se puede apreciar, el costo del diagnóstico precoz en niños de 5 y 12 años para la provincia de Nuble sería de \$155.371.406 (US aproximado: 315.000 a la fecha de publicación del artículo), para un total de 9.042 niños en total, siendo el costo de diagnóstico por niño de \$17.183 (US aproximado: 35).

\section{Discusión}

La alta morbilidad en ciertas regiones del país, la potencial letalidad en caso de no ser tratada oportunamente, la mayor frecuencia del diagnóstico en adultos jóvenes, los cuales se encuentran en la edad de máxima productividad laboral, y los costos por concepto de diagnóstico y tratamiento (hospitalizaciones y cirugías) incluyendo las recidivas, destacan la importancia epidemiológica de la hidatidosis. Además, esta zoonosis, por ser prevalente en zonas agrícolas y ganaderas, representa un importante problema de salud pública ${ }^{12}$.

Según la FAO (2007), si el paciente es intervenido quirúrgicamente, el costo de dicha intervención, en base a los datos existentes, se estima en U\$ 4.440; mientras que si el paciente recibe tratamiento con medicamentos tiene un costo estimado de U\$ 1.400 dólares, esto tomando en cuenta cinco ciclos de 30 días con cinco comprimidos diarios de albendazol. Estos datos son coincidentes con los obtenidos de la República Argentina que estiman un costo de tratamiento médico de U\$1.350 por paciente.
Si lo extrapolamos a los datos obtenidos en este estudio volvemos a corroborar la importancia económica y de la patología misma que tiene, el realizar un diagnóstico precoz de la enfermedad.

Para la realización de un diagnóstico precoz en Chile, en base a lo realizado en este estudio, para niños de 5 años es de U\$38 por persona y para niños de 12 años es de U\$26, aproximadamente. Por otro lado, en el caso de presentarse esta patología en un adulto, la intervención quirúrgica, hospitalización y exámenes realizados para un paciente con hidatidosis sería aproximadamente de U\$10.484 por persona.

Tabla 3. Costo del transporte de las muestras de suero para diagnóstico de hidatidosis (en pesos chilenos)

\begin{tabular}{|c|c|c|c|c|c|}
\hline Localidad & $\begin{array}{l}\text { Recurso } \\
\text { humano }\end{array}$ & $\begin{array}{c}\text { Equipos, } \\
\text { instrumentos } \\
\text { y muebles }\end{array}$ & Insumos & Alimentación & Total \\
\hline Cobquecura & 21.473 & 4.624 & 24.280 & 11.229 & 61.606 \\
\hline Quirihue & 16.568 & 3.742 & 18.455 & 8.664 & 47.429 \\
\hline Ninhue & 13.189 & 3.134 & 14.758 & 6.897 & 37.978 \\
\hline San Nicolás & 10.028 & 2.566 & 11.093 & 5.244 & 28.931 \\
\hline Chillán Viejo & 7.085 & 2.036 & 8.083 & 3.705 & 20.909 \\
\hline Chillán & 6.976 & 2.017 & 8.050 & 3.648 & 20.691 \\
\hline San Carlos & 10.355 & 2.624 & 11.764 & 5.415 & 30.158 \\
\hline Ñiquén & 13.407 & 3.173 & 15.396 & 7.011 & 38.987 \\
\hline San Fabián & 16.459 & 3.722 & 18.439 & 8.607 & 47.227 \\
\hline Coihueco & 10.464 & 2.644 & 11.780 & 5.472 & 30.360 \\
\hline Pinto & 10.137 & 2.585 & 11.715 & 5.301 & 29.738 \\
\hline San Ignacio & 10.900 & 2.722 & 12.468 & 5.700 & 31.790 \\
\hline El Carmen & 12.535 & 3.016 & 14.021 & 6.555 & 36.127 \\
\hline Yungay & 16.241 & 3.683 & 18.390 & 8.493 & 46.807 \\
\hline Pemuco & 13.189 & 3.134 & 14.758 & 6.897 & 37.978 \\
\hline Bulnes & 10.246 & 2.605 & 11.731 & 5.358 & 29.940 \\
\hline Quillón & 12.862 & 3.075 & 14.676 & 6.726 & 37.339 \\
\hline Ránquil & 13.625 & 3.212 & 15.445 & 7.125 & 39.407 \\
\hline Portezuelo & 12.099 & 2.938 & 13.924 & 6.327 & 35.288 \\
\hline Coelemu & 18.748 & 4.134 & 21.303 & 9.804 & 53.989 \\
\hline Trehuaco & 18.966 & 4.173 & 21.351 & 9.918 & 54.408 \\
\hline
\end{tabular}

Tabla 4. Costo total estimado del diagnóstico precoz de hidatidosis en niños de 5 años (en pesos chilenos)

Cohorte de niños de 5 años Costo de exámenes Total

Provincia de Ñuble 4.139 20.710 85.718 .690 
Tabla 5. Gastos totales de trasporte por ciudad (en pesos chilenos)

\begin{tabular}{|lccccc|}
\hline & $\begin{array}{c}\text { Extracción } \\
\text { de sangre }\end{array}$ & $\begin{array}{c}\text { Ecoto- } \\
\text { mografía }\end{array}$ & Transporte & $\begin{array}{c}\text { ELISA } \\
\text { indirecto }\end{array}$ & Total \\
\hline Chillán & 1.557 & 2.205 & 862 & 8.926 & 13.550 \\
\hline San Carlos & 1.557 & 2.205 & 1.257 & 8.926 & 13.945 \\
\hline Niquén & 1.557 & 2.205 & 1.695 & 8.926 & 14.383 \\
\hline San Fabián & 1.557 & 2.205 & 2.147 & 8.926 & 14.835 \\
\hline Coihueco & 1.557 & 2.205 & 1.265 & 8.926 & 13.953 \\
\hline Pinto & 1.557 & 2.205 & 1.239 & 8.926 & 13.927 \\
\hline San Ignacio & 1.557 & 2.205 & 1.325 & 8.926 & 14.013 \\
\hline Ránquil & 1.557 & 2.205 & 1.713 & 8.926 & 14.401 \\
\hline Portezuelo & 1.557 & 2.205 & 1.534 & 8.926 & 14.222 \\
\hline Coelemu & 1.557 & 2.205 & 2.571 & 8.926 & 15.259 \\
\hline Quillón & 1.557 & 2.205 & 1.623 & 8.926 & 14.311 \\
\hline Yungay & 1.557 & 2.205 & 2.128 & 8.926 & 14.816 \\
\hline Pemuco & 1.557 & 2.205 & 1.651 & 8.926 & 14.339 \\
\hline Bulnes & 1.557 & 2.205 & 1.248 & 8.926 & 13.936 \\
\hline Cobquecura & 1.557 & 2.205 & 2.934 & 8.926 & 15.622 \\
\hline Quirihue & 1.557 & 2.205 & 2.156 & 8.926 & 14.844 \\
\hline Ninhue & 1.557 & 2.205 & 1.651 & 8.926 & 14.339 \\
\hline San Nicolás & 1.557 & 2.205 & 1.206 & 8.926 & 13.894 \\
\hline Chillán Viejo & 1.557 & 2.205 & 871 & 8.926 & 13.559 \\
\hline El Carmen & 1.557 & 2.205 & 1.571 & 8.926 & 14.259 \\
\hline Trehuaco & 1.557 & 2.205 & 2.591 & 8.926 & 15.279 \\
\hline & & & & & \\
\hline
\end{tabular}

Tabla 6. Costo estimado del diagnóstico precoz de hidatidosis en niños de 12 años entre los años 2006 y 2007 (en pesos chilenos)

\begin{tabular}{|c|c|c|c|}
\hline $\begin{array}{l}\text { Provincia de } \\
\text { Ñuble }\end{array}$ & $\begin{array}{c}\text { Niños de } 12 \\
\text { años }\end{array}$ & $\begin{array}{l}\text { Costo de } \\
\text { examen }\end{array}$ & Total \\
\hline Chillán & 421 & 13.550 & 5.704 .550 \\
\hline San Carlos & 623 & 13.945 & 8.687 .735 \\
\hline Ñiquén & 353 & 14.383 & 5.077 .199 \\
\hline San Fabián & 63 & 14.835 & 934.605 \\
\hline Coihueco & 510 & 13.953 & 7.116 .030 \\
\hline Pinto & 181 & 13.927 & 2.520 .787 \\
\hline San Ignacio & 392 & 14.013 & 5.493 .096 \\
\hline El Carmen & 275 & 14.259 & 3.921 .225 \\
\hline Yungay & 171 & 14.816 & 2.533 .536 \\
\hline Pemuco & 187 & 14.339 & 2.681 .393 \\
\hline Bulnes & 313 & 13.936 & 4.361 .968 \\
\hline Quillón & 201 & 14.311 & 2.876 .511 \\
\hline Ránquil & 128 & 14.401 & 1.843 .328 \\
\hline Portezuelo & 118 & 14.222 & 1.678 .196 \\
\hline Coelemu & 191 & 15.259 & 2.914 .469 \\
\hline Trehuaco & 112 & 15.279 & 1.711 .248 \\
\hline Cobquecura & 138 & 15.622 & 2.155 .836 \\
\hline Quirihue & 108 & 14.844 & 1.603 .152 \\
\hline Ninhue & 138 & 14.399 & 1.987 .062 \\
\hline San Nicolás & 162 & 13.894 & 2.250 .828 \\
\hline Chillán Viejo & 118 & 13.559 & 1.599 .962 \\
\hline Total (\$) & & & 69.652 .716 \\
\hline
\end{tabular}

Tabla 7. Costos totales del diagnóstico precoz de hidatidosis (en pesos chilenos)

Provincia de Nuble

$\begin{array}{lr}\text { Niños de } 5 \text { años } & 85.718 .690 \\ \text { Niños de } 12 \text { años } & 69.652 .716 \\ \text { Total } & \$ 155.371 .406^{*}\end{array}$

*Aproximadamente US 315.000 al momento de publicarse este artículo.

La utilidad y la validez del diagnóstico precoz, ya sea por medio de ecotomografía o ELISA indirecto de la hidatidosis humana, han sido ampliamente verificadas tanto en pacientes sintomáticos como en asintomáticos ${ }^{12-15}$.

A lo mencionado anteriormente, se constata la importancia del estudio; es evidente la factibilidad de realizar un diagnóstico precoz de la enfermedad, así como también es de vital importancia, la utilización de un instrumento útil para tener certeza de los costos que genera esta enfermedad y así tomar algún tipo de decisión según el criterio económico expuesto en este estudio.

Se ha señalado en otras investigaciones, que el tratamiento de elección de la hidatidosis es la cirugía ${ }^{2,7,12}$, pero como se ha mencionado en esta investigación-todos los costos económicos que involucra- es bueno tener una alternativa al tratamiento quirúrgico que, en este caso, sería el uso de albendazol, tratamiento efectivo, ambulatorio, de fácil administración y económicamente accesible para los pacientes. Noemí y cols ${ }^{12}$, lo recomiendan como alternativa a la terapia para casos en que los quistes hidatídicos sean de difícil alcance quirúrgico o se encuentren en etapa inicial. De no poder realizarse un tratamiento no invasor también con este estudio se estaría promoviendo la cirugía temprana, que tiene mejor pronóstico quirúrgico por la minimización de las complicaciones; por ende, con el diagnóstico temprano 
se logra disminuir los tiempos de internación y las tasas de mortalidad.

Los costos que produce el diagnóstico precoz son bajos respecto a los costos que genera la patología; se puede evaluar como alternativa a los programas que están establecidos en Chile como, por ejemplo, los que van dirigidos al animal, para así poder atacar ambos lados de esta enfermedad (humano y animal). Los programas que se realizan hoy en día en Chile no rinden los frutos esperados, lo que se traduce en las ya mencionadas altas prevalencias de hidatidosis en algunas regiones del país ${ }^{2,17,18}$; por tanto, la alternativa propuesta de tamizaje precoz de la población escolar ayudaría a que las personas de zonas rurales no disminuyan su calidad de vida. También al realizar un diagnóstico precoz se estaría indirectamente informando sobre la patología, tanto a madres en los consultorios como a los niños en los establecimientos educacionales.

\section{Conclusiones}

Esta investigación surgió de la necesitad de determinar con claridad los costos implicados en la prevención de esta patología, particularmente en las personas más vulnerables a la infección por hidatidosis, como son los niños de zonas rurales, en forma de aportar datos concretos que apoyen un programa de salud pública contra esta patología.

Es de vital importancia tratar esta enfermedad tempranamente debido a las grandes complicaciones que origina, tanto a las personas afectadas como a las entidades de salud.

Para crear un programa sobre hidatidosis es necesario estar en conocimiento de los costos que ocasiona la prevención de esta dolencia. Como se ha mencionado anteriormente, para el correcto control de esta patología se utilizan métodos que van dirigidos a los animales y a la educación sanitaria de personas de zonas rurales, lo que se traduce en un buen manejo de animales de compañía y animales de abasto, o sea modificar los hábitos y costumbres de la comunidad, los que facilitan la propagación de la enfermedad. El gran problema radica en la falta de compromiso de ambas partes (entidades de salud y población de zonas rurales), debido a que en la gran mayoría de las personas se entera de la patología sólo cuando algún pariente o ellas mismas se habían contagiado, producto de la falta de información sobre la trasmisión de esta parasitosis desde los animales de compañía a los humanos.

La importancia de este estudio radica en que los controles que se han realizado hasta la actualidad no son fructíferos para las personas implicadas y para las entidades de salud, que no han visto la real magnitud del problema.

Por último, el resultado más relevante de esta investigación es que el costo total real para el diagnóstico precoz por niño es bajo y permite estar acorde con la política de salud de buscar la prevención más que el llegar al tratamiento de la dolencia.

\section{Resumen}

Se efectuó un análisis de costo de las diferentes intervenciones en el diagnóstico precoz de la hidatidosis, durante los años 2006 -2007 en la Provincia de Ñuble, Región del Bío-Bío, Chile. Esta investigación tenía por objetivo estimar los costos del diagnóstico precoz de esta patología; para ello se incluyó a todo niño de 5 y 12 años de zonas rurales de la provincia. Para la recolección de datos se determinaron las actividades, que consistieron en: medición de anticuerpos específicos en suero (ELISA indirecto) y la realización de una ecotomografía abdominal. En la determinación de costos se identificaron los costos reales directos e indirectos incurridos mediante la metodología de costos basados en actividades. Los resultado obtenidos demuestran que el diagnóstico preventivo implica un gasto por niño de $\$ 17.183$; por ende, realizar esta intervención es de menor costo que esperar a que la persona se enferme de dicha patología.

\section{Referencias}

1.- Aliaga F, Oberg C. Epidemiología de la hidatidosis humana en la IX Región de la Araucanía, Chile: 1991-1998. Bol Chil Parasitol 2000; 55 (3-4): 54-8.

2.- Lorca M, Díaz X, Segura J, Álvarez C. Estudio de prevalencia serológica de hidatidosis en caprinos de Til Til y Colina, Santiago de Chile 2005. Revista electrónica de veterinaria REDVET 2006; 7 (12): 1-6. http://www.veterinaria.org/revistas/redvet/ n121206/120611.pdf [consulta: 19 enero 2008].
3.- Cortés S, Valle C. Hidatidosis humana: Generalidades y situación epidemiológica en Chile según egresos hospitalarios y notificación obligatoria entre los años 2001 y 2005. Rev Chil Infect 2010; 27(4): 329-35.

4.- OPS/OMS. Informe del Proyecto Subregional Cono Sur de Control y Vigilancia de la Hidatidosis Argentina, Brasil, Chile y Uruguay (Primera reunión constitutiva). Montevideo, Uruguay. 2004; 1-123. http://www.bvsops.org. uy/pdf/equinoc.pdf [consulta: 15 noviembre 2010].

5.- Legua P. Hidatidosis. Rev Med Hered 2002; 13 (3): 77-8.
6.- Castro T. Prevalencia de hidatidosis en bovinos faenados durante el año 2003 en el frigorífico Temuco S.A. y su impacto en la salud humana, IX Región. Memoria de título Médico Veterinario, Universidad Católica de Temuco, Facultad de Acuicultura y Ciencias Veterinarias, Temuco, Chile. 2004.

7.- Núñez E, Calero D, Estares L, Morales A. Prevalencia y factores de riesgo de hidatidosis en población general del distrito de Ninacaca-Pasco, Perú 2001. An Fac Med 2003; 64 (1): 34-42.

8.- FAO. Estimación del impacto económico de la equinococosis quística en el cono sur 
(Argentina, Brasil, Chile y Uruguay). 2007; 1-20. http://www.paho.org/spanish/AD/DPC/ VP/hidatidosis-impacto-econ-07fao. pdf [consulta: 15 diciembre 2008].

9.- Larrieu E, Belloto A, Arambulo P, Tamayo H. Echinococcosis quística: epidemiología y control en América del Sur. Parasitol Latinoam 2004; 59: 82-9.

10.- Ribal M, Lidid L, López B, Werner A, Zulantay I, De Diego P. Hidatidosis múltiple. Parasitol Día 2000; 24 (1-2): 46-8.

11.- Vera G, Venturelli F, Ramírez J, Venturelli A. Hidatidosis humana. Cuad Cir 2003; 17 (1): 88-94.
12.- Noemí I, Viovy A, Zamorano R, Blanco A, Revello D, Vojkovic M, et al. Hidatidosis en la infancia: Albendazol en su tratamiento médico y quirúrgico. Rev Chil Infect 2003; 20 (4): 229-34.

13.- Larrieu E, Dapcich C, Guarnera E, Coltorti E, Bianchi C, Moguilansky A. Evaluación de ELISA y DD5 en el diagnóstico de la hidatidosis humana en población asintomática. Rev San Hig Púb 1994; 68 (3): 393-8.

14.- Larrieu E, Frider B, Del Carpio M, Salvitti J, Mercapide C, Pereyra R, et al. Portadores asintomáticos de hidatidosis: epidemiología, diagnóstico y tratamiento. Pan Am J Public Health 2000; 8 (4): 250-6.

15.- Perdomo R, Parada R, Álvarez C, Cattivelli D, Geninazzi H, Barragué A, et al. Estudio epidemiológico de hidatidosis. Detección precoz por ultrasonido en áreas de alto riesgo. Rev Med Urug 1990; 6 (1): 34-47.

16.- Butte J, Mege R, Caraccil M, Báez S, Díaz A, Viñuela E, et al. Hidatidosis alveolar hepática. Rev Chil Cir 2004; 56 (6): 593-7.

17.- Neira P, Subercaseaux B, De la Rosa A, Rusowsky L. Hidatidosis hepato-pulmonar en una preescolar, caso clínico. Rev Chil Pediatr 2006; 77 (2): 169-76. 